\title{
TSIRELSON LIKE OPERATOR SPACES
}

\author{
HUN HEE LEE
}

\begin{abstract}
We construct nontrivial examples of weak- $C_{p}(1 \leq p \leq \infty)$ operator spaces with the local operator space structure very close to $C_{p}=[R, C]_{\frac{1}{p}}$. These examples are non-homogeneous Hilbertian operator spaces, and their constructions are similar to that of 2-convexified Tsirelson's space by W. B. Johnson.
\end{abstract}

\section{INTRODUCTION}

Tsirelson's space and its variations have been sources of counterexamples to many questions in the Banach space theory (See 1 for the details). In this paper we focus on the 2-convexified version of Tsirelson's space, which served as an example of nontrivial weak Hilbert space, a closest object to Hilbert spaces in the sense of type and cotype theory.

Recall that a Banach space $X$ is called a weak Hilbert space (6]) if for any $0<\delta<1$ there is a constant $C>0$ with the following property : for any finite dimensional $F \subseteq X$ we can find $F_{1} \subseteq F$ and an onto projection $P: X \rightarrow F_{1}$ satisfying

$$
d_{F_{1}}:=d\left(F_{1}, \ell_{2}^{\operatorname{dim} F_{1}}\right) \leq C, \operatorname{dim} F_{1} \geq \delta \operatorname{dim} F \text { and }\|P\| \leq C,
$$

where $d(\cdot, \cdot)$ is the Banach-Mazur distance defined by

$$
d(X, Y)=\inf \left\{\|u\|\left\|u^{-1}\right\| \mid u: X \rightarrow Y, \text { isomorphism }\right\} .
$$

As an operator space analogue of weak Hilbert space the author introduced the notion of weak- $H$ spaces for a (separable and infinite dimensional) perfectly Hilbertian operator space $H$ in [5]. A Hilbertian operator space $H$ (i.e. $H$ is isometric to a Hilbert space) is called homogeneous if for every $u: H \rightarrow H$ we have $\|u\|_{c b}=\|u\|$ and subquadratic if for all orthogonal projections $\left\{P_{i}\right\}_{i=1}^{n}$ in $H$ with $I_{H}=P_{1}+\cdots+P_{n}$ we have

$$
\|x\|_{B\left(\ell_{2}\right) \otimes_{\min } H}^{2} \leq \sum_{i=1}^{n}\left\|I_{B\left(\ell_{2}\right)} \otimes P_{i}(x)\right\|_{B\left(\ell_{2}\right) \otimes_{\min } H}^{2}
$$

for any $x \in B\left(\ell_{2}\right) \otimes H$ (See p.82 of [9]), where $\otimes_{\min }$ is the injective tensor product of operator spaces. A homogeneous Hilbertian operator space $H$ is called perfectly Hilbertian if $H$ and $H^{*}$ is subquadratic. See [4] for the definition of weak- $H$ space and the related type and cotype notions of operator spaces.

In [5] it is shown that an operator space $E$ is a weak- $H$ space if and only if for any $0<\delta<1$ there is a constant $C>0$ with the following property : for any finite dimensional $F \subseteq E$ we can find $F_{1} \subseteq F$ and an onto projection $P: E \rightarrow F_{1}$ satisfying

$$
d_{F_{1}, c b}^{H}:=d_{c b}\left(F_{1}, H_{\operatorname{dim} F_{1}}\right) \leq C, \operatorname{dim} F_{1} \geq \delta \operatorname{dim} F \text { and }\|P\|_{c b} \leq C,
$$

where $d_{c b}(\cdot, \cdot)$ is the cb-distance defined by

$$
d_{c b}(E, F)=\inf \left\{\|u\|_{c b}\left\|u^{-1}\right\|_{c b} \mid u: E \rightarrow F, \text { isomorphism }\right\}
$$

Key words and phrases. cotype, operator space, weak Hilbert space, Tsirelson's space. 2000 Mathematics Subject Classification. Primary 47L25, Secondary 46B07. 
and $H_{n}$ is the $n$-dimensional subspace of $H$. Thus, we can say that weak- $H$ spaces have similar local operator space structure to $H$.

The aim of this paper is to construct nontrivial examples of weak- $H$ spaces (not completely isomorphic to $H$ ) for $H=C_{p}, R_{p}$ and $1 \leq p \leq \infty$, where $C_{p}=[C, R]_{\frac{1}{p}}$ and $R_{p}=[R, C]_{\frac{1}{p}}$, interpolation spaces of the column and the row Hilbert space via complex method. We will follow the approach of W. B. Johnson (and T. Figiel) to construct Hilbertian operator spaces $X_{C_{p}}$ and $X_{R_{p}}$ for $1 \leq p \leq \infty$, Since $R_{p}^{*}=C_{p^{\prime}}$ for $\frac{1}{p}+\frac{1}{p^{\prime}}=1$ it is enough to consider $X_{C_{p}}$ and $X_{R_{p}}$ for $1 \leq p \leq 2$. Interestingly, the construction for the case $1 \leq p<2$ and the case $p=2$ are different although many proofs are overlapping.

In section 2 we prepare some back ground materials concerning vector valued Schatten classes and a description of $d_{E, c b}^{H}$. In section 3 we focus on $1 \leq p<2$ case. In section 3.1 we will construct $X_{C_{p}}$ (resp. $X_{R_{p}}$ ) and investigate the behavior of its canonical basis. It will be shown that the span of certain block sequences of the canonical basis is completely isomorphic to $C_{p}$ (resp. $R_{p}$ ) of the same dimension with bounded constants. In section 3.2 we will examine that $X_{C_{p}}\left(\right.$ resp. $X_{R_{p}}$ ) is our desired space. First, we show that $X_{C_{p}}$ (resp. $X_{R_{p}}$ ) is a weak- $C_{p}$ (resp. weak- $\left.R_{p}\right)$. We prepare additional materials concerning $\pi_{2, H}$-norms, operator space analogue of absolutely 2-summing norm, and related description of $d_{E, c b}^{H}$. Secondly, it will be shown that $X_{C_{p}}$ (resp. $X_{R_{p}}$ ) is not completely isomorphic to $C_{p}$ (resp. $R_{p}$ ) investigating containment of an isomorphic copy of $c_{0}$ (the Banach space of all sequences vanishing at infinity) as in chapter 13 of [7].

In section 4 we consider $p=2$ case. Most of arguments from section 3 are still available with some exceptions. In the final remark we construct a non-Hilbertian example of weak- $\mathrm{OH}$ space.

Throughout this paper, we assume that the reader is familiar with basic concepts in Banach spaces $(2,7,11)$ and operator spaces (3, 10). In this paper $E$ and $H$ will be reserved for an operator space and a separable, infinite dimensional and perfectly Hilbertian operator space. Note that $H(I)$ is well-defined for any index set $I\left([8)\right.$. We will simply write $H_{n}$ when $I=\{1, \cdots, n\}$. As usual, $B(E, F)$ and $C B(E, F)$ denote the set of all bounded linear maps and all cb-maps from $E$ into $F$, respectively.

$\mathcal{K}$ implies the algebra of compact operators on $\ell_{2}$ and $\mathcal{K}_{0}$ is the union of the increasing sequence $M_{1} \subseteq \cdots \subseteq M_{n} \subseteq M_{n+1} \subseteq \cdots$ of matrix algebras. Note that $\mathcal{K}=\overline{\mathcal{K}_{0}}$.

\section{Preiminaries}

In this section we collect some back ground materials which will be used later.

2.1. Vector valued Schatten classes. Let $S_{p}(\mathcal{H})$ be the Schatten class on a Hilbert space $\mathcal{H}$ and $1 \leq p \leq \infty$. In [9] $S_{p}(\mathcal{H}, E), E$-valued Schatten classes are defined by

$$
S_{p}(\mathcal{H} ; E):=\left[S_{\infty}(\mathcal{H}) \otimes_{\min } E, S_{1}(\mathcal{H}) \widehat{\otimes} E\right]_{\frac{1}{p}},
$$

where $\otimes_{\min }$ and $\widehat{\otimes}$ refer to injective and projective tensor products of operator spaces. When $\mathcal{H}=\ell_{2}$ we simply write as $S_{p}$ and $S_{p}(E)$.

The above vector valued Schatten classes are useful to describe operator space structure of subspaces of $S_{p}$. In particular, the operator space structure of $C_{p}, R_{p}$ and $\ell_{p}\left(c_{0}\right.$ when $\left.p=\infty\right)$ can be described as follows.

$$
\left\|\sum_{i} x_{i} \otimes e_{i}\right\|_{S_{p}\left(C_{p}\right)}=\left\|\left(\sum_{i} x_{i}^{*} x_{i}\right)^{\frac{1}{2}}\right\|_{S_{p}},
$$




$$
\left\|\sum_{i} x_{i} \otimes e_{i}\right\|_{S_{p}\left(R_{p}\right)}=\left\|\left(\sum_{i} x_{i} x_{i}^{*}\right)^{\frac{1}{2}}\right\|_{S_{p}}
$$

and

$$
\left\|\sum_{i} x_{i} \otimes e_{i}\right\|_{S_{p}\left(\ell_{p}\right)}=\left(\sum_{i}\left\|x_{i}\right\|_{S_{p}}^{p}\right)^{\frac{1}{p}} .
$$

When we are dealing with $E+{ }_{p} F$, the sum of operator spaces in the sense of $\ell_{p}$, it is more appropriate to use vector valued Schatten classes. See chapter 2 of $[9]$ for the definition of $E \oplus_{p} F$, the direct sum of operator spaces in the sense of $\ell_{p}$. Then we define $E+{ }_{p} F:=\left(E \oplus_{p} F\right) /(E \cap F)$. Then we have

$$
S_{p}\left(E \oplus_{p} F\right)=S_{p}(E) \oplus_{p} S_{p}(F) \text { and } S_{p}\left(E+{ }_{p} F\right)=S_{p}(E)+_{p} S_{p}(F) .
$$

Moreover, we can use vector valued Schatten classes to check complete boundedness. Indeed, by Lemma 1.7. of 9 for any cb-map $T: E \rightarrow F$ between operator spaces we have

$$
\|T\|_{c b}=\sup _{n \geq 1}\left\|I_{S_{p}^{n}} \otimes T: S_{p}^{n}(E) \rightarrow S_{p}^{n}(F)\right\| .
$$

For a subspace $S \subseteq S_{p}$ we denote $S(E):=\overline{\operatorname{span}}\{S \otimes E\} \subseteq S_{p}(E)$. We will focus on the case $S=C_{p}, R_{p}$ and $\ell_{p}\left(c_{0}\right.$ when $\left.p=\infty\right)$. By Theorem 1.1. of [9] we have

$$
S_{p}(E) \cong C_{p} \otimes_{h} E \otimes_{h} R_{p}
$$

completely isometrically by the identification $e_{i j} \otimes x \mapsto e_{i 1} \otimes x \otimes e_{1 j}$, so that $C_{p}(E)$ and $R_{p}(E)$ are 1-completely complemented in $S_{p}(E)$.

By taking the natural diagonal projection $\ell_{p}(E)$ is also 1-completely complemented in $S_{p}(E)$, and we have

$$
\ell_{p}(E)=\left[c_{0} \otimes_{\min } E, \ell_{1} \widehat{\otimes} E\right]_{\frac{1}{p}} .
$$

By the above observations for any cb-map $T: E \rightarrow F$ between operator spaces we have

$$
\left\|I_{S} \otimes T: S(E) \rightarrow S(F)\right\|_{c b}=\|T\|_{c b}
$$

for $S=C_{p}, R_{p}$ and $\ell_{p}\left(c_{0}\right.$ when $\left.p=\infty\right)$.

2.2. A description of $d_{E, c b}^{H}$ and operator spaces with similar $n$-dimensional structure to $H$. In this section we present several observation concerning $(2, H)$ summing maps, operator space analogues of 2-summing maps, and related description of $d_{E, c b}^{H}$.

For a linear map $T: E \rightarrow F$ between operator maps the $(2, H)$-summing norm $\pi_{2, H}(T)$ is defined by

$$
\pi_{2, H}(T):=\sup \left\{\frac{\left(\sum_{k}\left\|T S e_{k}\right\|^{2}\right)^{\frac{1}{2}}}{\left\|S: H^{*} \rightarrow E\right\|_{c b}}\right\} .
$$

We need the subquadracity of $H$ to ensure that $\pi_{2, H}(\cdot)$ is a norm.

The factorization norm through $H$ of $T, \gamma_{H}(T)$, is defined by

$$
\gamma_{H}(T):=\inf \left\{\left\|T_{1}\right\|_{c b}\left\|T_{2}\right\|_{c b}\right\},
$$

where the infimum runs over all possible factorization

$$
T: E \stackrel{T_{1}}{\longrightarrow} H^{*}(I) \stackrel{T_{2}}{\longrightarrow} F
$$

for some index set $I$. Note that the trace dual $\gamma_{H}^{*}$ of $\gamma_{H}$ can be described as follows (Theorem 6.1. of [8]). For any finite rank map $T: E \rightarrow F$ we have

$$
\gamma_{H}^{*}(T)=\inf \left\{\pi_{2, H^{*}}\left(T_{1}\right) \pi_{2, H}\left(T_{2}^{*}\right)\right\}
$$


where the infimum runs over all possible factorization

$$
T: E \stackrel{T_{1}}{\longrightarrow} \ell_{2}^{m} \stackrel{T_{2}}{\longrightarrow} F
$$

for some $m \in \mathbb{N}$.

We need to consider completely nuclear norm $\nu^{o}(\cdot)([3])$, which is the trace dual of cb-norm $\|\cdot\|_{c b}$. By arguments in p.200-201 of [3] we have

$$
|\operatorname{tr}(T)| \leq \nu^{o}(T)
$$

for all finite rank map $T: E \rightarrow E$.

The following Lemma is an operator space version of the fact that the composition of two 2-summing maps is a nuclear map in some special cases.

Lemma 2.1. Let $H$ be a subquadratic, homogeneous and Hilbertian operator space and $E$ be a finite dimensional operator space. Then for any $u: E \rightarrow E$ we have

$$
\nu^{o}(u) \leq \inf \left\{\pi_{2, H}(S) \pi_{2, H}^{*}(T)\right\},
$$

where the infimum runs over all possible factorization

$$
u: E \stackrel{S}{\rightarrow} \ell_{2}^{n} \stackrel{T}{\rightarrow} E .
$$

Proof. Let $u: E \rightarrow E$ and consider any factorization $u: E \stackrel{S}{\rightarrow} \ell_{2}^{n} \stackrel{T}{\rightarrow} E$. Then for any $v: E \rightarrow E$ and any further factorization $T: \ell_{2}^{n} \stackrel{A}{\rightarrow} H^{*} \stackrel{B}{\rightarrow} E$ we have

$$
\begin{aligned}
|\operatorname{tr}(v u)| & =|\operatorname{tr}(v B A S)|=|\operatorname{tr}(S v B A)| \leq\|A\|_{H S}\|S v B\|_{H S} \\
& =\|A\|_{H S} \pi_{2, H}(S v B) \leq\|A\|_{H S}\|B\|_{c b}\|v\|_{c b} \pi_{2, H}(S) .
\end{aligned}
$$

By taking infimum over all possible $A, B, S$ and $v$ with $\|v\|_{c b} \leq 1$ we get the desired result using trace duality.

Now we present a description of $d_{E, c b}^{H}$ using $(2, H)$-summing norms.

Lemma 2.2. Let $H$ be a perfectly Hilbertian operator space. Then we have

$$
d_{E, c b}^{H}=\sup \left\{\frac{\pi_{2, H^{*}}^{*}(u)}{\pi_{2, H}\left(u^{*}\right)} \mid u: \ell_{2}^{m} \rightarrow E, m \in \mathbb{N}\right\} .
$$

Proof. We get the result form Theorem 4.3. of [4] and the fact that

$$
\pi_{2, H}\left(u^{*}\right) \leq \ell(u) \leq \pi_{2, H^{*}}^{*}(u)
$$

for any $u: \ell_{2}^{m} \rightarrow F$. later.

We show an operator space version of Remark 13.4. of [8], which will be useful

Proposition 2.3. Let $H$ be a perfectly Hilbertian operator space and $n \in \mathbb{N}$ be fixed. Suppose that $E$ is an operator space satisfying the following: there is a constant $C>0$ such that for any $n$-dimensional subspace $F \subseteq E$ we have

$$
d_{F, c b}^{H} \leq C .
$$

Then for any $n$-dimensional subspace $F \subseteq E$ we have a projection $P: E \rightarrow E$ onto F with

$$
\gamma_{H}(P) \leq C .
$$

Proof. By combining Lemma 2.2 and the assumption we get

$$
\pi_{2, H^{*}}^{*}(T) \leq C \cdot \pi_{2, H}\left(T^{*}\right)
$$

for any $T: \ell_{2}^{n} \rightarrow E$.

Now we fix a $n$-dimensional subspace $F \subseteq E$ and let $i: F \hookrightarrow E$ be the inclusion. For any $u: F \rightarrow F$ we consider a factorization

$$
i u: F \stackrel{\alpha}{\rightarrow} \ell_{2}^{m} \stackrel{\beta}{\rightarrow} E .
$$


Then by applying Lemma 2.1, (2.4) and (2.5) we have

$$
\begin{aligned}
|\operatorname{tr}(u)| & \leq \nu^{o}(u) \leq \pi_{2, H^{*}}(\alpha) \pi_{2, H^{*}}^{*}\left(\left.\beta\right|_{\operatorname{ran} \alpha}\right) \\
& \leq C \cdot \pi_{2, H^{*}}(\alpha) \pi_{2, H}\left(\left(\left.\beta\right|_{\operatorname{ran} \alpha}\right)^{*}\right) \\
& \leq C \cdot \pi_{2, H^{*}}(\alpha) \pi_{2, H}\left(\beta^{*}\right) .
\end{aligned}
$$

By taking infimum over all possible $\alpha$ and $\beta$ we get

$$
|\operatorname{tr}(u)| \leq C \cdot \gamma_{H}^{*}(i u)
$$

If we apply Hahn-Banach theorem to the functional $u \mapsto \operatorname{tr}(u)$ we get the desired result.

\section{THE CASE $1 \leq p<2$}

3.1. The construction and basic properties of the canonical basis. In this section we will construct Hilbertian operator spaces $X_{C_{p}}$ and $X_{R_{p}}$ for $1 \leq p<2$, consequently $X_{C_{p}}$ and $X_{R_{p}}$ for $1 \leq p \leq \infty, p \neq 2$. We will mainly focus on $X_{C_{p}}$ case only since the situation of $X_{R_{p}}$ is symmetric.

We say that a disjoint collection, $\left(E_{j}\right)_{j=1}^{f(k)}$, of finite subsets of $\mathbb{N}$ is "allowable" if

$$
E_{j} \subseteq\{k, k+1, \cdots\} \text { for all } 1 \leq j \leq f(k),
$$

where $k \in \mathbb{N}$ and $f(k)=\left(4 k^{3}\right)^{k}$. This specific choice of $f$ will be clarified later in section 4.2. For a finite subset $E \subseteq \mathbb{N}$ and

$$
x=\sum_{i \geq 1} x_{i} \otimes t_{i} \in \mathcal{K}_{0} \otimes c_{00},
$$

where $t_{i}$ is the $i$-th unit vector in $c_{00}$ (finitely supported sequences of complex numbers), we denote

$$
E x=\sum_{i \in E} x_{i} \otimes t_{i}
$$

Let $1 \leq p<2$ and $0<\theta<1$ be fixed. We will define a sequence of norms on $\mathcal{K}_{0} \otimes c_{00}$ to construct $X_{C_{p}}$ (resp. $X_{R_{p}}$ ). For $x \in \mathcal{K}_{0} \otimes c_{00}$ we define

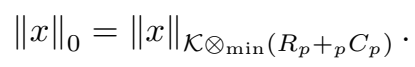

Then $X_{0}$, the completion of $\left(c_{00},\|\cdot\|_{0}\right)$, is nothing but the homogeneous Hilbertian operator space $R_{p}+{ }_{p} C_{p}$, and clearly $\|\cdot\|_{0}$ satisfies Ruan's axioms. Now we define $\left(\|\cdot\|_{n}\right)_{n \geq 0}$ recursively. Suppose that $\|\cdot\|_{n}$ is already defined and satisfies Ruan's axioms $([3,10])$. Then $X_{n}$, the completion of $\left(c_{00},\|\cdot\|_{n}\right)$ is an operator space, and we define

$$
\begin{array}{r}
\|x\|_{n+1}=\max \left\{\|x\|_{n}, \theta \sup \left\|\sum_{j=1}^{f(k)} e_{j 1} \otimes E_{j} x\right\|_{\mathcal{K} \otimes_{\min } C_{p}\left(X_{n}\right)}\right\} \\
\left(\text { resp. } \max \left\{\|x\|_{n}, \theta \sup \left\|\sum_{j=1}^{f(k)} e_{1 j} \otimes E_{j} x\right\|_{\mathcal{K} \otimes_{\min } R_{p}\left(X_{n}\right)}\right\}\right),
\end{array}
$$

where the inner supremum runs over all "allowable" sequence $\left\{E_{j}\right\}_{j=1}^{f(k)} \subseteq \mathbb{N}$. Then $\|\cdot\|_{n+1}$ satisfies Ruan's axioms, so that $X_{n+1}$, the completion of $\left(c_{00},\|\cdot\|_{n+1}\right)$ is an operator space. Actually, $X_{n+1}$ is a subspace of $X_{n} \oplus_{\infty} \ell_{\infty}\left(I ;\left\{C_{p}\left(X_{n}\right)\right\}\right)$ spanned by elements of the form, $\left(x,\left(\theta E_{j} x\right)_{\left(E_{j}\right) \in I}\right)$, where $I$ is the collection of all allowable sequences, so that $X_{n+1}$ inherits the operator space structure from $X_{n} \oplus_{\infty}$ $\ell_{\infty}\left(I ;\left\{C_{p}\left(X_{n}\right)\right\}\right)$ (the case for $R_{p}$ is similar).

Remark 3.1. When we write $e_{j 1} \otimes E_{j} x \in \mathcal{K} \otimes_{\min } C_{p}\left(X_{n}\right)$ one should note that $e_{j 1} \in C_{p}$ and $E_{j} x \in \mathcal{K} \otimes X_{n}$, which is twisted in order. 
Proposition 3.2. For any $x \in \mathcal{K}_{0} \otimes c_{00},\left(\|x\|_{n}\right)_{n \geq 0}$ is increasing, and we have

$$
\|x\|_{\mathcal{K} \otimes_{\min }\left(R_{p}+{ }_{p} C_{p}\right)} \leq\|x\|_{n} \leq\|x\|_{\mathcal{K} \otimes_{\min } C_{p}}
$$

for all $n \geq 0$

Proof. The left inequality is clear. For the right inequality we use induction on $n$. When $n=0$, it is trivial. Suppose we have the right inequality for $n$ and for all $x \in \mathcal{K}_{0} \otimes c_{00}$, equivalently, the formal identity $C_{p} \rightarrow X_{n}$ is completely contractive. Then we have

$$
\begin{aligned}
\theta\left\|\sum_{j=1}^{f(k)} e_{j 1} \otimes E_{j} x\right\|_{S_{p}\left(C_{p}\left(X_{n}\right)\right)} & =\theta\left\|\sum_{j=1}^{f(k)} \sum_{i \in E_{j}} x_{i} \otimes e_{i} \otimes e_{j 1}\right\|_{S_{p}\left(C_{p}\left(X_{n}\right)\right)} \\
& \leq \theta\left\|\sum_{j=1}^{f(k)} \sum_{i \in E_{j}} x_{i} \otimes e_{i 1} \otimes e_{j 1}\right\|_{S_{p}\left(C_{p}\left(C_{p}\right)\right)} \\
& =\theta\left\|\left(\sum_{j=1}^{f(k)} \sum_{i \in E_{j}} x_{i}^{*} x_{i}\right)^{\frac{1}{2}}\right\| \\
& \leq \theta\|x\|_{S_{p}\left(C_{p}\right)}<\|x\|_{S_{p}\left(C_{p}\right)} .
\end{aligned}
$$

Thus, we have that

$$
x \mapsto \theta \sum_{j=1}^{f(k)} e_{j 1} \otimes E_{j} x, C_{p} \rightarrow C_{p}\left(X_{n}\right)
$$

is a complete contraction, and so is

$$
x \mapsto x \oplus \theta \sum_{j=1}^{f(k)} e_{j 1} \otimes E_{j} x, C_{p} \rightarrow X_{n} \oplus_{\infty} C_{p}\left(X_{n}\right)
$$

by the assumption, which leads us to the right inequality for $n+1$ and for all $x \in \mathcal{K}_{0} \otimes c_{00}$.

Now we can consider $\|x\|=\lim _{n \rightarrow \infty}\|x\|_{n}$ for all $x \in \mathcal{K}_{0} \otimes c_{00}$, and clearly $\|\cdot\|$ satisfies Ruan's axioms, so that $X_{C_{p}}$ (resp. $\left.X_{R_{p}}\right)$, the completion of $\left(c_{00},\|\cdot\|\right)$ is an operator space. Actually, $X_{C_{p}}$ (resp. $\left.X_{R_{p}}\right)$ is a subspace of $\ell_{\infty}\left(X_{n}\right)$ spanned by elements of the form, $(x, x, \cdots, x)$, so that $X_{C_{p}}$ (resp. $\left.X_{R_{p}}\right)$ inherits the operator space structure from $\ell_{\infty}\left(X_{n}\right)$. Moreover, $X_{C_{p}}\left(\right.$ resp. $\left.X_{R_{p}}\right)$ is Hilbertian by Proposition 3.2

We have a slight different form of $\|\cdot\|_{n}$ which will be useful later.

Proposition 3.3. For any $x \in \mathcal{K}_{0} \otimes c_{00}$ and any $n \geq 0$ we have

$$
\|x\|_{n+1}=\max \left\{\|x\|_{0}, \theta \sup \left\|\sum_{j=1}^{f(k)} e_{j 1} \otimes E_{j} x\right\|_{\mathcal{K} \otimes_{\min } C_{p}\left(X_{n}\right)}\right\},
$$

where the inner supremum runs over all "allowable" sequence $\left\{E_{j}\right\}_{j=1}^{f(k)} \subseteq \mathbb{N}$.

Proof. Suppose we have

$$
\|x\|_{n+1}>\theta \sup \left\{\left\|\sum_{j=1}^{f(k)} e_{j 1} \otimes E_{j} x\right\|_{\mathcal{K} \otimes_{\min } C_{p}\left(X_{n}\right)}\right\}
$$


for an $x \in \mathcal{K}_{0} \otimes c_{00}$. Then by the definition of $\|\cdot\|_{n+1}$ we have $\|x\|_{n+1}=\|x\|_{n}$. Since the formal identity $i_{n}: X_{n} \rightarrow X_{n-1}$ is clearly a complete contraction we get another complete contraction

$$
I_{C_{p}} \otimes i_{n}: C_{p}\left(X_{n}\right) \rightarrow C_{p}\left(X_{n-1}\right)
$$

by (2.3). Thus, it follows that

$$
\begin{array}{r}
\|x\|_{n}>\theta \sup \left\{\left\|\sum_{j=1}^{f(k)} e_{j 1} \otimes E_{j} x\right\|_{\mathcal{K} \otimes_{\min } C_{p}\left(X_{n}\right)}\right\} \\
\geq \theta \sup \left\{\left\|\sum_{j=1}^{f(k)} e_{j 1} \otimes E_{j} x\right\|_{\mathcal{K} \otimes_{\min } C_{p}\left(X_{n-1}\right)}\right\}
\end{array}
$$

and hence $\|x\|_{n}=\|x\|_{n-1}$. If we repeat this process, then we get $\|x\|_{n+1}=\|x\|_{0}$. if

We say that a basis $\left\{f_{i}\right\}_{i \geq 1}$ of an operator space $E$ is $C$-completely unconditional

$$
\left\|\sum_{i \geq 1} a_{i} x_{i} \otimes f_{i}\right\|_{\mathcal{K} \otimes_{\min } E} \leq C\left\|\sum_{i \geq 1} x_{i} \otimes f_{i}\right\|_{\mathcal{K} \otimes_{\min } E}
$$

for any finitely supported sequence of $\left\{x_{i}\right\}_{i \geq 1} \subseteq \mathcal{K}_{0}$ and any sequence of scalars $\left(a_{i}\right)_{i \geq 1}$ with $\left|a_{i}\right| \leq 1$ for all $i \geq 1$.

Proposition 3.4. The canonical basis $\left\{t_{i}\right\}_{i \geq 1}$ is a normalized 1-completely unconditional basis for $X_{C_{p}}$.

Proof. We will use induction on $n$, to show that $\left\{t_{i}\right\}_{i \geq 1}$ is a normalized 1-completely unconditional basis for $X_{n}$. First, we fix a sequence $\left(a_{i}\right)_{i \geq 1}$ with $\left|a_{i}\right| \leq 1$ for all $i \geq 1$.

When $n=0$, for any $\sum_{i \geq 1} x_{i} \otimes e_{i} \in \mathcal{K}_{0} \otimes c_{00}$ we have

$$
\begin{aligned}
& \left\|\sum_{i \geq 1} a_{i} x_{i} \otimes t_{i}\right\|_{S_{p}\left(R_{p}+{ }_{p} C_{p}\right)}^{p} \\
& =\inf _{x_{i}=y_{i}+z_{i}}\left\{\left\|\left(\sum_{i \geq 1}\left|a_{i}\right|^{2} y_{i} y_{i}^{*}\right)^{\frac{1}{2}}\right\|_{S_{p}}^{p}+\left\|\left(\sum_{i \geq 1}\left|a_{i}\right|^{2} z_{i}^{*} z_{i}\right)^{\frac{1}{2}}\right\|_{S_{p}}^{p}\right\} \\
& \leq \inf _{x_{i}=y_{i}+z_{i}}\left\{\left\|\left(\sum_{i \geq 1} y_{i} y_{i}^{*}\right)^{\frac{1}{2}}\right\|_{S_{p}}^{p}+\left\|\left(\sum_{i \geq 1} z_{i}^{*} z_{i}\right)^{\frac{1}{2}}\right\|_{S_{p}}^{p}\right\} \\
& =\left\|\sum_{i \geq 1} x_{i} \otimes t_{i}\right\|_{S_{p}\left(R_{p}+{ }_{p} C_{p}\right)}^{p},
\end{aligned}
$$

since $\left|a_{i}\right|^{2} y_{i} y_{i}^{*} \leq y_{i} y_{i}^{*}$ and $\left|a_{i}\right|^{2} z_{i}^{*} z_{i} \leq z_{i}^{*} z_{i}$.

Suppose we have the result for $n$, which is equivalent to

$$
X_{n} \rightarrow X_{n}, t_{i} \mapsto a_{i} t_{i}
$$

is a complete contraction. Then by (2.3) we have another complete contraction

$$
C_{p}\left(X_{n}\right) \rightarrow C_{p}\left(X_{n}\right), e_{j 1} \otimes t_{i} \mapsto e_{j 1} \otimes a_{i} t_{i} .
$$


Thus, for any "allowable" sequence $\left\{E_{j}\right\}_{j=1}^{f(k)} \subseteq \mathbb{N}$ we have

$$
\begin{aligned}
& \theta\left\|\sum_{j=1}^{f(k)} e_{j 1} \otimes E_{j}\left(\sum_{i} a_{i} x_{i} \otimes t_{i}\right)\right\|_{\mathcal{K} \otimes \min }\left(C_{p}\left(X_{n}\right)\right) \\
& =\theta\left\|\sum_{j=1}^{f(k)} \sum_{i \in E_{j}} x_{i} \otimes e_{j 1} \otimes a_{i} t_{i}\right\|_{\mathcal{K} \otimes_{\min }\left(C_{p}\left(X_{n}\right)\right)}\|\|_{\mathcal{K} \otimes_{\min }\left(C_{p}\left(X_{n}\right)\right)} \leq\left\|\sum_{j=1} x_{i} \otimes t_{i}\right\|_{n+1}, \\
& \leq \theta E_{j},
\end{aligned}
$$

which implies the result for $n+1$.

Now we investigate the operator space structure of the subspace spanned by certain normalized and disjoint block sequences of $\left\{t_{i}\right\}_{i \geq 1} \subseteq X_{C_{p}}$. They are $\theta$ completely isomorphic to $C_{p}$ with the same dimension.

Proposition 3.5. Let $\left(y_{j}\right)_{j=1}^{f(k)}$ be a disjoint and normalized block sequences of $\left\{t_{i}\right\}_{i \geq 1} \subseteq X_{C_{p}}$ with supp $y_{j} \subseteq\{k, k+1, \cdots\}$ for $1 \leq j \leq f(k)$. Then we have

$$
\theta\left\|\sum_{j=1}^{f(k)} b_{j} \otimes e_{j 1}\right\|_{S_{p}\left(C_{p}\right)} \leq\left\|\sum_{j=1}^{f(k)} b_{j} \otimes y_{j}\right\|_{S_{p}\left(X_{C_{p}}\right)} \leq\left\|\sum_{j=1}^{f(k)} b_{j} \otimes e_{j 1}\right\|_{S_{p}\left(C_{p}\right)}
$$

for any $\left(b_{j}\right)_{j=1}^{f(k)} \subseteq S_{p}$.

Proof. For the left inequality we set $E_{j}=\operatorname{supp} y_{j}$ and $n_{j}=\min \left\{\operatorname{supp} y_{j}\right\}$. Since

$$
X_{C_{p}} \rightarrow C_{p}\left(X_{n}\right), x \mapsto \theta \sum_{j=1}^{f(k)} e_{j 1} \otimes E_{j} x
$$

is a complete contraction we have

$$
\begin{aligned}
\left\|\sum_{i=1}^{f(k)} b_{i} \otimes y_{i}\right\|_{S_{p}\left(X_{C_{p}}\right)} & \geq \theta\left\|\sum_{j=1}^{f(k)} e_{j 1} \otimes E_{j}\left(\sum_{i=1}^{f(k)} b_{i} \otimes y_{i}\right)\right\|_{S_{p}\left(C_{p}\left(X_{n}\right)\right)} \\
& =\theta\left\|\sum_{j=1}^{f(k)} b_{j} \otimes e_{j 1} \otimes y_{j}\right\|_{S_{p}\left(C_{p}\left(X_{n}\right)\right)} \\
& \geq \theta\left\|\sum_{j=1}\right\| f(k) \\
& \geq \theta\left\|\sum_{j=1} \otimes e_{j 1} \otimes t_{n_{j}}\right\|_{S_{p}\left(C_{p}\left(X_{n}\right)\right)} \otimes e_{j 1} \|_{S_{p}\left(C_{p}\right)}
\end{aligned}
$$

by Lemma 3.6 and 3.7 below for any $\left(b_{i}\right)_{j=1}^{f(k)} \subseteq S_{p}$. 
For the right inequality we will show the following. For any disjoint and normalized sequence $\left(y_{j}\right)_{j=1}^{f(k)} \subseteq X_{C_{p}}$ we have

$$
\left\|\sum_{j=1}^{f(k)} b_{j} \otimes y_{j}\right\|_{S_{p}\left(X_{n}\right)} \leq\left\|\sum_{j=1}^{f(k)} b_{j} \otimes e_{j 1}\right\|_{S_{p}\left(C_{p}\right)}
$$

for all $\left(b_{j}\right)_{j=1}^{f(k)} \subseteq S_{p}$.

Let us use induction on $n$. When $n=0$ we are done since

$$
C B\left(C_{p}, R_{p}+{ }_{p} C_{p}\right)=B\left(C_{p}, R_{p}+{ }_{p} C_{p}\right)
$$

isometrically and $\left(e_{j 1}\right)_{j \geq 1}$ and $\left(y_{j}\right)_{j=1}^{f(k)}$ are orthonormal. Suppose we have (3.1) for $n$. Then for any "allowable" sequence $\left\{E_{j}\right\}_{j=1}^{f(l)} \subseteq \mathbb{N}$ we have

$$
\begin{aligned}
& \theta\left\|\sum_{j=1}^{f(l)} e_{j 1} \otimes E_{j}\left(\sum_{i=1}^{f(k)} b_{i} \otimes y_{i}\right)\right\|_{S_{p}\left(C_{p}\left(X_{n}\right)\right)} \\
& =\left\|\sum_{j=1}^{f(l)} \sum_{i=1}^{f(k)} b_{i} \otimes e_{j 1} \otimes \theta E_{j} y_{i}\right\|_{S_{p}\left(C_{p}\left(X_{n}\right)\right)} \\
& \leq\left\|\sum_{j=1}^{f(l)} \sum_{i=1}^{f(k)} b_{i} \otimes e_{j 1} \otimes\right\| \theta E_{j} y_{i}\left\|e_{1, i j}\right\|_{S_{p}\left(C_{p}\left(C_{p}\right)\right)} \leq\left\|\left(\sum_{i=1}^{f(k)} b_{i}^{*} b_{i}\right)^{\frac{1}{2}}\right\|_{S_{p}} . \\
& =\left\|\left(\sum_{j=1}^{f(l)} \sum_{i=1}^{f(k)} b_{i}^{*} b_{i}\left\|\theta E_{j} y_{i}\right\|^{2}\right)^{\frac{1}{2}}\right\|_{S_{p}} \leq \|
\end{aligned}
$$

The last inequality comes from

$$
\sum_{j=1}^{f(l)}\left\|\theta E_{j} y_{i}\right\|^{2} \leq \theta^{2}\left\|y_{i}\right\|^{2} \leq 1 .
$$

This conclude (3.1) for $n+1$ as before.

Lemma 3.6. Let $\left(y_{j}\right)_{j=1}^{f(k)},\left(E_{j}\right)_{j=1}^{f(k)}$ and $\left(n_{j}\right)_{j=1}^{f(k)}$ be the same as in Proposition 3.5. Then we have

$$
\left\|\sum_{j=1}^{f(k)} b_{j} \otimes t_{n_{j}}\right\|_{S_{p}\left(X_{n}\right)} \leq\left\|\sum_{j=1}^{f(k)} b_{j} \otimes y_{j}\right\|_{S_{p}\left(C_{p}\right)}
$$

for all $\left(b_{j}\right)_{j=1}^{f(k)} \subseteq S_{p}$.

Proof. We use induction on $n$. When $n=0$ we are done since $R_{p}+{ }_{p} C_{p}$ is a homogeneous Hilbertian operator space and $\left(t_{n_{j}}\right)_{j=1}^{f(k)}$ and $\left(y_{j}\right)_{j=1}^{f(k)}$ are orthonormal. Suppose we have (3.2) for $n$, and consider any fixed "allowable" sequence $\left\{F_{j}\right\}_{j=1}^{f(k)} \subseteq$ $\mathbb{N}$. If we set

$$
x=\sum_{j=1}^{f(k)} b_{j} \otimes t_{n_{j}}, y=\sum_{j=1}^{f(k)} b_{j} \otimes y_{j}
$$

and

$$
G_{j}=\left\{n_{i}: n_{i} \in F_{j}\right\} \text { for } 1 \leq j \leq f(k),
$$

then

$$
G_{j} y=\sum_{n_{i} \in F_{j}} b_{i} \otimes t_{n_{i}}
$$


and $\left\{G_{j}\right\}_{j=1}^{f(k)}$ is an "allowable" sequence. Consequently, we have

$$
\begin{aligned}
\theta\left\|\sum_{j=1}^{f(k)} e_{j 1} \otimes F_{j} x\right\|_{S_{p}\left(C_{p}\left(X_{n}\right)\right)} & =\theta\left\|\sum_{j=1}^{f(k)} \sum_{n_{i} \in F_{j}} b_{i} \otimes e_{j 1} \otimes t_{n_{i}}\right\|_{S_{p}\left(C_{p}\left(X_{n}\right)\right)} \\
& =\theta\left\|\sum_{j=1}^{f(k)} e_{j 1} \otimes G_{j} y\right\|_{S_{p}\left(C_{p}\left(X_{n}\right)\right)} \\
& \leq\|y\|_{S_{p}\left(X_{n+1}\right)} .
\end{aligned}
$$

The last line is obtained by the complete contraction

$$
X_{n+1} \rightarrow C_{p}\left(X_{n}\right), z \mapsto \theta \sum_{j=1}^{f(k)} e_{j 1} \otimes G_{j} z .
$$

This conclude (3.2) for $n+1$ as before.

Lemma 3.7. We have

$$
\left\|\sum_{j \geq 1} b_{j} \otimes e_{j 1} \otimes t_{j}\right\|_{S_{p}\left(C_{p}\left(X_{n}\right)\right)} \geq\left\|\sum_{j \geq 1} b_{j} \otimes e_{j 1}\right\|_{S_{p}\left(C_{p}\right)}
$$

for all finitely supported $\left(b_{j}\right)_{j \geq 1} \subseteq S_{p}$.

Proof. Consider a finitely supported $\left(b_{j}\right)_{j \geq 1} \subseteq S_{p}$. Then we have

$$
\left\|\sum_{j \geq 1} b_{j} \otimes e_{j 1} \otimes t_{j}\right\|_{S_{p}\left(C_{p}\left(X_{n}\right)\right)} \geq\left\|\sum_{j \geq 1} b_{j} \otimes e_{j 1} \otimes e_{j}\right\|_{S_{p}\left(C_{p}\left(R_{p}+_{p} C_{p}\right)\right)} .
$$

Note that $C_{p}\left(R_{p}+{ }_{p} C_{p}\right) \cong C_{p}\left(R_{p}\right)+{ }_{p} C_{p}\left(C_{p}\right) \cong S_{p}+{ }_{p} C_{p}$ completely isometrically. Since the formal identities $\ell_{1} \rightarrow C_{1}$ and $\ell_{2} \rightarrow C_{2}$ are complete contractions so is $\ell_{p} \rightarrow C_{p}$ by complex interpolation. Since $\overline{\operatorname{span}}\left\{e_{j 1} \otimes e_{j}\right\}_{j \geq 1}$ correspond to $\ell_{p}$ and $C_{p}$ in $S_{p}$ and $C_{p}\left(C_{p}\right)$, respectively, we have

$$
\overline{\operatorname{span}}\left\{e_{j 1} \otimes e_{j}\right\}_{j \geq 1}\left(\subseteq C_{p}\left(R_{p}+{ }_{p} C_{p}\right)\right) \cong C_{p}
$$

completely isometrically. Thus, we have

$$
\left\|\sum_{j \geq 1} b_{j} \otimes e_{j 1} \otimes t_{j}\right\|_{S_{p}\left(C_{p}\left(X_{n}\right)\right)}=\left\|\sum_{j \geq 1} b_{j} \otimes e_{j 1}\right\|_{S_{p}\left(C_{p}\right)} .
$$

\section{2. $X_{C_{p}}$ is a nontrivial weak- $C_{p}$ space.}

Proposition 3.8. For $n \in \mathbb{N}$ we consider

$$
Y_{n}=\overline{\operatorname{span}}\left\{t_{i}\right\}_{i \geq n+1} \subseteq X_{C_{p}} .
$$

Then for any $E \subseteq Y_{n}$ with $\operatorname{dim} E=n$, we have

$$
d_{c b}\left(E, C_{p}^{n}\right) \leq 3 \theta^{-1} .
$$

Proof. By Proposition V.6 of [1 there is a linear map $V: E \rightarrow Y_{n}$ such that $V(E) \subseteq \overline{\operatorname{span}}\left\{y_{i}\right\}_{i=1}^{f(n)}$, where $y_{i}$ 's are disjoint elements in $Y_{n}$ and

$$
\|V f-f\| \leq \frac{1}{2 n}\|f\|
$$


for all $f \in E$. Now we consider the Auerbach basis $\left(x_{i}, x_{i}^{*}\right)_{i=1}^{n}$ of $E$. Then we have

$$
\sum_{i=1}^{3 n}\left\|x_{i}^{*}\right\|\left\|x_{i}-V x_{i}\right\| \leq \frac{1}{2 n} \sum_{i=1}^{n}\left\|x_{i}\right\| \leq \frac{1}{2}
$$

which implies

$$
d_{c b}(E, V(E)) \leq 3
$$

by the perturbation lemma (Lemma 2.13.2 of [10]). On the other hand we have

$$
d_{c b}\left(\overline{\operatorname{span}}\left\{y_{i}\right\}_{i=1}^{f(n)}, C_{p}^{f(n)}\right) \leq \theta^{-1}
$$

by Proposition 3.5. Consequently, by combining these we get our desired result.

Remark 3.9. The employment of Proposition V.6 of [1] in the proof of Proposition 3.8 is the reason why we have chosen $f(k)=\left(4 k^{3}\right)^{k}$.

Actually we can show that every $n$-dimensional subspace of $Y_{n}$ in Proposition 3.8 is completely complemented with bounded constants, so that we are ready to prove one of our main results.

Theorem 3.10. $X_{C_{p}}$ is a weak- $C_{p}$ space.

Proof. Let $Y_{n}$ be the same as in Proposition 3.8. Consider any $E \subseteq X_{C_{p}}$ with

$$
\operatorname{dim} E=2 n \text { or } 2 n+1 .
$$

Then we have $\operatorname{dim}\left(E \cap Y_{n}\right) \geq n$, so that there is $F \subseteq E$ such that $\operatorname{dim} F=n$ and $F \subseteq Y_{n}$. Then by Proposition 3.8 we have

$$
d_{F, c b}^{H} \leq 3 \theta^{-1} .
$$

Moreover, $F$ is $3 \theta^{-1}$-completely complemented in $Y_{n}$ by Proposition 2.3. Since $Y_{n}$ itself is 1-completely complemented in $X_{C_{p}}$ so is $F$, which implies $X_{C_{p}}$ is a weak- $C_{p}$ space.

All we have to do now is to show that $X_{C_{p}}$ is not completely isomorphic to $C_{p}$. Before that we need to prepare the following lemmas which are analogues of Lemma 13.6 and 13.7 in [7.

Lemma 3.11. Let $N \in \mathbb{N}$ be fixed. Then, for any $n \geq 0$ and any $y, z \in C_{p} \otimes X_{C_{p}}$ with

$$
\operatorname{supp} y \subseteq\{1,2, \cdots, N\} \text { and } \operatorname{supp} z \subseteq\{N+1, N+2, \cdots\}
$$

we have

$$
\|y+z\|_{C_{p} \otimes_{\min } X_{n+1}} \leq \max \left\{\|y\|_{C_{p} \otimes_{\min } X_{n+1}}+\alpha\|z\|_{C_{p} \otimes_{\min } X_{n}},\|z\|_{C_{p} \otimes_{\min } X_{n+1}}\right\},
$$

where $\alpha=\max \{1, \theta f(N)\}$.

Proof. Let us fix $n \geq 0$ and $y, z \in C_{p} \otimes X_{C_{p}}$ with

$$
\operatorname{supp} y \subseteq\{1,2, \cdots, N\} \text { and } \operatorname{supp} z \subseteq\{N+1, N+2, \cdots\} .
$$

Let $T^{y}, T^{z}$ and $T^{y+z}$ are linear maps from $R_{p}$ into $X_{C_{p}}$ associated with $y, z$ and $y+z$, respectively.

Then we have

$$
\|y+z\|_{C_{p} \otimes_{\min } X_{n+1}}=\left\|T^{y+z}: R_{p} \rightarrow X_{n+1}\right\|_{c b} .
$$

For any $x=\sum_{i} x_{i} \otimes e_{1 i} \in \mathcal{K} \otimes R_{p}$ we consider $\left\|T^{y+z}(x)\right\|_{n+1}$. If

$$
\left\|T^{y+z}(x)\right\|_{n+1}=\left\|T^{y+z}(x)\right\|_{n},
$$


then we have

$$
\begin{aligned}
\left\|T^{y+z}(x)\right\|_{n+1} & \leq\left\|T^{y}(x)\right\|_{n}+\left\|T^{z}(x)\right\|_{n} \\
& \leq\left(\left\|T^{y}: R_{p} \rightarrow X_{n}\right\|_{c b}+\left\|T^{z}: R_{p} \rightarrow X_{n}\right\|_{c b}\right)\|x\|_{\mathcal{K} \otimes R_{p}} \\
& \leq\left(\|y\|_{C_{p} \otimes_{\min } X_{n+1}}+\|z\|_{C_{p} \otimes_{\min } X_{n}}\right)\|x\|_{\mathcal{K} \otimes R_{p}} .
\end{aligned}
$$

If not, we consider any "allowable" sequence $\left\{E_{j}\right\}_{j=1}^{f(k)} \subseteq \mathbb{N}$. When $k>N$, we have

$$
\begin{aligned}
\theta\left\|\sum_{j=1}^{f(k)} e_{j 1} \otimes E_{j}\left[T^{y+z}(x)\right]\right\|_{\mathcal{K} \otimes_{\min } C_{p}\left(X_{n}\right)} & =\theta\left\|\sum_{j=1}^{f(k)} e_{j 1} \otimes E_{j}\left[T^{z}(x)\right]\right\|_{\mathcal{K} \otimes_{\min } C_{p}\left(X_{n}\right)} \\
& \leq\left\|T^{z}(x)\right\|_{n+1} \leq\|z\|_{C_{p} \otimes_{\min } X_{n+1}}\|x\|_{\mathcal{K} \otimes R_{p}} .
\end{aligned}
$$

Otherwise, we have

$$
\begin{aligned}
& \theta\left\|\sum_{j=1}^{f(k)} e_{j 1} \otimes E_{j}\left[T^{y+z}(x)\right]\right\|_{\mathcal{K} \otimes_{\min } C_{p}\left(X_{n}\right)} \\
& =\theta\left\|\sum_{j=1}^{f(k)} e_{j 1} \otimes E_{j}\left[T^{y}(x)\right]\right\|_{\mathcal{K} \otimes_{\min } C_{p}\left(X_{n}\right)}+\theta\left\|\sum_{j=1}^{f(k)} e_{j 1} \otimes E_{j}\left[T^{z}(x)\right]\right\|_{\mathcal{K} \otimes_{\min } C_{p}\left(X_{n}\right)} \\
& \leq\left\|T^{y}(x)\right\|_{n+1}+\theta \sum_{j=1}^{f(k)}\left\|E_{j}\left[T^{z}(x)\right]\right\|_{n} \\
& \leq\left(\|y\|_{C_{p} \otimes_{\min } X_{n+1}}+\theta N\|z\|_{C_{p} \otimes_{\min } X_{n}}\right)\|x\|_{\mathcal{K} \otimes R_{p}} .
\end{aligned}
$$

Combining the above results we get our desired estimate.

Lemma 3.12. For any $n \geq 0$ and any $x \in \mathcal{K} \otimes_{\min } X_{C_{p}}$ we have

$$
\|x\|_{\mathcal{K} \otimes_{\min } X_{C_{p}}} \leq\left\|\left(x, \theta^{n} x\right)\right\|_{\mathcal{K} \otimes_{\min }\left(X_{n} \oplus p C_{p}\right)}
$$

Proof. We will use induction on $n$ to show (3.3) for all $x \in \mathcal{K}_{0} \otimes c_{00}$.

When $n=0$, it is trivial. Suppose we have (3.3) for all $x \in \mathcal{K}_{0} \otimes c_{00}$ and for a fixed $n \geq 0$, which is equivalent to the fact that the map

$$
\left(x, \theta^{n} x\right) \mapsto x, F \rightarrow X_{C_{p}}
$$

is a complete contraction, where $F=\overline{\operatorname{span}}\left\{\left(y, \theta^{n} y\right): y \in c_{00}\right\} \subseteq X_{n} \oplus_{p} C_{p}$. Then, for any "allowable" sequence $\left\{E_{j}\right\}_{j=1}^{f(k)} \subseteq \mathbb{N}$ we have another complete contraction

$$
e_{j 1} \otimes\left(x, \theta^{n} x\right) \mapsto e_{j 1} \otimes x, C_{p}(F) \rightarrow C_{p}\left(X_{C_{p}}\right)
$$


Thus, we have

$$
\begin{aligned}
& \theta\left\|\sum_{j=1}^{f(k)} e_{j 1} \otimes E_{j} x\right\|_{\mathcal{K} \otimes_{\min } C_{p}\left(X_{C_{p}}\right)} \\
& \leq\left\|\sum_{j=1}^{f(k)} e_{j 1} \otimes\left(\theta E_{j} x, \theta^{n+1} E_{j} x\right)\right\|_{\mathcal{K} \otimes_{\min } C_{p}\left(X_{n} \oplus_{p} C_{p}\right)} \\
& =\left\|\left(\sum_{j=1}^{f(k)} e_{j 1} \otimes \theta E_{j} x, \sum_{j=1}^{f(k)} e_{j 1} \otimes \theta^{n+1} E_{j} x\right)\right\|_{\mathcal{K} \otimes_{\min }\left[C_{p}\left(X_{n}\right) \oplus_{p} C_{p}\left(C_{p}\right)\right]} \\
& \leq\left\|\left(x, \theta^{n+1} x\right)\right\|_{\mathcal{K} \otimes_{\min }\left(X_{n+1} \oplus_{p} C_{p}\right)}
\end{aligned}
$$

The last line follows from the fact that the maps

$$
x \mapsto \theta \sum_{j=1}^{f(k)} e_{j 1} \otimes E_{j} x, X_{n+1} \rightarrow C_{p}\left(X_{n}\right)
$$

and

$$
x \mapsto \sum_{j=1}^{f(k)} e_{j 1} \otimes E_{j} x, C_{p} \rightarrow C_{p}\left(C_{p}\right)
$$

are complete contractions.

Since

$$
\|x\|_{m+1}=\max \left\{\|x\|_{0}, \theta \sup \left\|\sum_{j=1}^{f(k)} e_{j 1} \otimes E_{j} x\right\|_{\mathcal{K} \otimes_{\min } C_{p}\left(X_{m}\right)}\right\}
$$

for all $m \geq 0$ by Proposition 3.3, we have

$$
\|x\| \leq \max \left\{\|x\|_{0}, \theta \sup \left\|\sum_{j=1}^{f(k)} e_{j 1} \otimes E_{j} x\right\|_{\mathcal{K} \otimes_{\min } C_{p}\left(X_{C_{p}}\right)}\right\},
$$

which leads us to our desired conclusion.

The following proposition is the crucial point to explain why we cannot have complete isomorphism between $C_{p}$ and $X_{C_{p}}$.

Proposition 3.13. Let $n \geq 0$. Then, $C_{p} \otimes_{\min } E$ contains an isomorphic copy of $c_{0}$ for any infinite dimensional subspace $E \subseteq X_{n}$.

Proof. We will use induction on $n$. Consider $n=0$. Since

$$
C_{p} \otimes_{\min }\left(R_{p}+{ }_{p} C_{p}\right) \hookrightarrow B\left(\ell_{2}\right), e_{j 1} \otimes e_{j} \mapsto e_{j j}
$$

$\left(e_{j 1} \otimes e_{j}\right)_{j \geq 1}$ is a basic sequence in $C_{p} \otimes_{\min }\left(R_{p}+{ }_{p} C_{p}\right)$ equivalent to the canonical basis of $c_{0}$. Since $R_{p}+_{p} C_{p}$ is homogeneous, every infinite dimensional subspace $E \subseteq R_{p}+{ }_{p} C_{p}$ is completely isometric to $R_{p}+{ }_{p} C_{p}$ itself. Thus, we get the desired result for $n=0$.

Now suppose that $C_{p} \otimes_{\min } E$ contains an isomorphic copy of $c_{0}$ for any infinite dimensional subspace $E \subseteq X_{n}$. Let $F \subseteq X_{n+1}$ be infinite dimensional, and $\epsilon>0$ be arbitrarily given. We claim that there is an infinite dimensional subspace $F^{\prime} \subseteq F$ such that $C_{p} \otimes_{\min } F^{\prime}$ and $\left.C_{p} \otimes_{\min } X_{n}\right|_{F^{\prime}}$ are isomorphic or we can choose a sequence $\left(x_{i}\right)_{i \geq 1} \subseteq C_{p} \otimes_{\min } F$ satisfying

$$
\left\|x_{i}\right\|_{C_{p} \otimes_{\min } X_{n+1}}=1
$$


for all $i \geq 1$ and

$$
\left\|\sum_{i=1}^{M} x_{i}\right\|_{C_{p} \otimes_{\min } X_{n+1}} \leq 1+\epsilon
$$

for all $M \geq 1$. Both cases imply $C_{p} \otimes_{\min } F$ contains an isomorphic copy of $c_{0}$, and consequently we get our desired induction result.

For the claim we start with a norm 1 vector $x_{1}=e_{11} \otimes x \in C_{p} \otimes_{\min } F$. Suppose we have disjoint and finitely supported $x_{1}, \cdots, x_{m} \in C_{p} \otimes_{\min } F$ with

$$
\left\|x_{i}\right\|_{C_{p} \otimes_{\min } X_{n+1}}=1
$$

for all $1 \leq i \leq m$ and

$$
\left\|\sum_{i=1}^{m} x_{i}\right\|_{C_{p} \otimes_{\min } X_{n+1}} \leq 1+\epsilon \sum_{i=1}^{m-1} \frac{1}{2^{i}} .
$$

Let $N$ be a natural number such that $N \geq \operatorname{supp} x_{i}$ for all $1 \leq i \leq m$ and

$$
Y_{N}=\overline{\operatorname{span}}\left\{e_{i}\right\}_{i \geq N+1} \subseteq X_{n+1} .
$$

If $C_{p} \otimes_{\min } F \cap Y_{N}$ and $\left.C_{p} \otimes_{\min } X_{n}\right|_{F \cap Y_{N}}$ are isomorphic, then it is done by the induction hypothesis. Suppose $C_{p} \otimes_{\min } F \cap Y_{N}$ and $\left.C_{p} \otimes_{\min } X_{n}\right|_{F \cap Y_{N}}$ are not isomorphic. Then there is a finitely supported

$$
x_{m+1} \in C_{p} \otimes_{\min } F \cap Y_{N} \text { with } \operatorname{supp} x_{m+1} \subseteq\{N+1, N+2, \cdots\}
$$

satisfying

$$
\left\|x_{m+1}\right\|_{C_{p} \otimes_{\min } X_{n+1}}=1 \text { and }\left\|x_{m+1}\right\|_{C_{p} \otimes_{\min } X_{n}}<\frac{\epsilon}{2^{m} \alpha},
$$

where $\alpha=\max \{1, \theta f(N)\}$. Then by Lemma 3.11 we have

$$
\left\|\sum_{i=1}^{m+1} x_{i}\right\|_{C_{p} \otimes_{\min } X_{n+1}} \leq 1+\epsilon \sum_{i=1}^{m} \frac{1}{2^{i}} .
$$

By repeating this process we get our claim.

Theorem 3.14. $X_{C_{p}}$ is not completely isomorphic to $C_{p}$.

Proof. Suppose that $X_{C_{p}}$ is $C$-completely isomorphic to $C_{p}$ for some $C>0$. Then $X_{C_{p}}$ is $(C+\epsilon)$-homogeneous for any $\epsilon>0$. Thus, by repeating the proof of Proposition 10.1 in [10] to $X_{C_{p}}$ and $C_{p}$ we get

$$
\|I\|_{c b}=\|I\|_{c b}\left\|I^{-1}\right\|_{c b} \leq(C+\epsilon)^{2} d_{c b}\left(X_{C_{p}}, C_{p}\right) \leq(C+\epsilon)^{3},
$$

where $I: X_{C_{p}} \rightarrow C_{p}$ is the formal identity. By Lemma 3.12 and (2.1) we have

$$
\begin{aligned}
(C+\epsilon)^{-3 p}\|x\|_{S_{p}\left(C_{p}\right)}^{p} & \leq\|x\|_{S_{p}\left(X_{C_{p}}\right)}^{p} \leq\left\|\left(x, \theta^{n} x\right)\right\|_{S_{p}\left(X_{n} \oplus_{p} C_{p}\right)}^{p} \\
& =\|x\|_{S_{p}\left(X_{n}\right)}^{p}+\theta^{p n}\|x\|_{S_{p}\left(C_{p}\right)}^{p}
\end{aligned}
$$

for any $x \in \mathcal{K}_{0} \otimes c_{00}$.

If we choose $n$ large enough so that $\theta^{p n}<\frac{(C+\epsilon)^{-3 p}}{2}$, then we get

$$
\frac{(C+\epsilon)^{-3 p}}{2}\|x\|_{S_{p}\left(C_{p}\right)}^{p} \leq\|x\|_{S_{p}\left(X_{n}\right)}^{p} .
$$

Consequently, we have

$$
d_{c b}\left(X_{n}, C_{p}\right) \leq \frac{(C+\epsilon)^{3}}{2^{\frac{1}{p}}}
$$

However, since $C_{p} \otimes_{\min } C_{p} \subseteq C B\left(R_{p}, C_{p}\right) \cong S_{\frac{2 p}{2-p}}$ isometrically and $S_{\frac{2 p}{2-p}}$ is a reflexive Banach space with a basis $C_{p} \otimes_{\min } X_{n}$ does not contain any isomorphic copy of $c_{0}$, which is contradictory to Proposition 3.13 . 
Remark 3.15. Note that if a weak- $H$ space is a homogeneous Hilbertian space, then it is completely isomorphic to $H$ itself by Proposition 3.8. of [4]. Thus, $X_{C_{p}}$ is not homogeneous.

\section{THE CASE $p=2$}

4.1. The construction and basic properties of the canonical basis. We will construct $\mathrm{X}_{\mathrm{OH}}$, an example of nontrivial weak-OH space, in a similar way. Many arguments used in section 3 still work for $O H$ case also, so that we only provide the proofs which we need to approach in a significantly different way.

Consider a fixed constant $0<\theta<1$. For $x \in \mathcal{K}_{0} \otimes c_{00}$ we define

$$
\|x\|_{0}=\|x\|_{\mathcal{K} \otimes_{\min }\left(\min \ell_{2}\right)}
$$

and for $n \geq 0$

$$
\|x\|_{n+1}=\max \left\{\|x\|_{n}, \theta \sup \left\|\left(E_{j} x\right)_{j=1}^{f(k)}\right\|_{\mathcal{K} \otimes_{\min } \ell_{2}^{f(k)}\left(X_{n}\right)}\right\},
$$

where the inner supremum runs over all "allowable" sequence $\left\{E_{j}\right\}_{j=1}^{f(k)} \subseteq \mathbb{N}$. As before we denote the completion of $\left(c_{00},\|\cdot\|_{n}\right)$ by $X_{n}$ and $X_{n+1}$ inherits the operator space structure from $X_{n} \oplus_{\infty} \ell_{\infty}\left(I ;\left\{\ell_{2}^{f(k)}\left(X_{n}\right)\right\}\right)$, where $I$ is the collection of all allowable sequences.

Proposition 4.1. For any $x \in \mathcal{K}_{0} \otimes c_{00},\left(\|x\|_{n}\right)_{n \geq 0}$ is increasing, and we have

for all $n \geq 0$.

$$
\|x\|_{\mathcal{K} \otimes_{\min }\left(\min \ell_{2}\right)} \leq\|x\|_{n} \leq\|x\|_{\mathcal{K} \otimes_{\min } O H}
$$

Now we can consider $\|x\|=\lim _{n \rightarrow \infty}\|x\|_{n}$ for all $x \in \mathcal{K}_{0} \otimes c_{00}$, and $X_{O H}$, the completion of $\left(c_{00},\|\cdot\|\right)$ inherits the operator space structure from $\ell_{\infty}\left(X_{n}\right)$. Moreover, $X_{O H}$ is Hilbertian by Proposition 4.1.

Proposition 4.2. For any $x \in \mathcal{K}_{0} \otimes c_{00}$ and any $n \geq 0$ we have

$$
\|x\|_{n+1}=\max \left\{\|x\|_{0}, \theta \sup \left\|\left(E_{j} x\right)_{j=1}^{f(k)}\right\|_{\mathcal{K} \otimes_{\min } \ell_{2}^{f(k)}\left(X_{n}\right)}\right\},
$$

where the inner supremum runs over all "allowable" sequence $\left\{E_{j}\right\}_{j=1}^{f(k)} \subseteq \mathbb{N}$.

Proposition 4.3. The canonical basis $\left\{t_{i}\right\}_{i \geq 1}$ is a normalized 1-completely unconditional basis for $X_{O H}$.

Proof. The only different point from the proof of Proposition 3.4 is the proof for $X_{0}$. Indeed, for any $\sum_{i \geq 1} x_{i} \otimes t_{i} \in \mathcal{K}_{0} \otimes c_{00}$ we have

$$
\begin{aligned}
\left\|\sum_{i \geq 1} a_{i} x_{i} \otimes t_{i}\right\|_{0} & =\left\|\sum_{i \geq 1} a_{i} x_{i} \otimes e_{i}\right\|_{\mathcal{K} \otimes_{\min } \min \ell_{2}}=\left\|u: \ell_{2} \rightarrow \mathcal{K}, e_{i} \mapsto a_{i} x_{i}\right\| \\
& =\sup \left\{\left\|\sum_{i \geq 1} \xi_{i} a_{i} x_{i}\right\|: \sum_{i \geq 1}\left|\xi_{i}\right|^{2} \leq 1\right\} \\
& \leq\left\|v: \ell_{2} \rightarrow \mathcal{K}, e_{i} \mapsto x_{i}\right\| \leq\left\|\sum_{i \geq 1} x_{i} \otimes t_{i}\right\|_{0}
\end{aligned}
$$

since $\sum_{i \geq 1}\left|\xi_{i} a_{i}\right|^{2} \leq 1$

Now we investigate operator space structure spanned by certain disjoint block sequences of $\left\{t_{i}\right\}_{i \geq 1}$. They are $\theta$-completely isomorphic to operator Hilbert spaces with the same dimensions. 
Proposition 4.4. Let $\left(y_{j}\right)_{j=1}^{f(k)}$ be a disjoint and normalized block sequence of $\left\{t_{i}\right\}_{i \geq 1}$ such that $\operatorname{supp}\left(y_{j}\right) \subseteq\{k, k+1, \cdots\}$ for all $1 \leq j \leq f(k)$. Then we have

$$
\theta\left(\sum_{j=1}^{f(k)}\left\|b_{j}\right\|_{S_{2}}^{2}\right)^{\frac{1}{2}} \leq\left\|\sum_{j=1}^{f(k)} b_{j} \otimes y_{j}\right\|_{S_{2}\left(X_{O H}\right)} \leq\left(\sum_{j=1}^{f(k)}\left\|b_{j}\right\|_{S_{2}}^{2}\right)^{\frac{1}{2}}
$$

Proof. Consider any "allowable" sequence $\left\{E_{j}\right\}_{j=1}^{f(k)} \subseteq \mathbb{N}$. Since we have

$$
X_{n+1} \rightarrow \ell_{2}^{f(k)}\left(X_{n}\right), x \mapsto\left(\theta E_{j} x\right)_{j=1}^{f(k)}
$$

is completely contractive

$$
X_{O H} \rightarrow \ell_{2}^{f(k)}\left(X_{n}\right), x \mapsto\left(\theta E_{j} x\right)_{j=1}^{f(k)}
$$

is also completely contractive. Thus if we set $E_{j}=\operatorname{supp}\left(y_{j}\right)$, then $\left\{E_{j}\right\}_{j=1}^{f(k)}$ is "allowable", so that we have

$$
\begin{aligned}
\left\|\sum_{j=1}^{f(k)} b_{j} \otimes y_{j}\right\|_{S_{2}\left(X_{O H}\right)} & \geq\left\|\left(\theta E_{j}\left(\sum_{i=1}^{f(k)} b_{i} \otimes y_{i}\right)\right)_{j=1}^{f(k)}\right\|_{S_{2}\left(\ell_{2}^{f(k)}\left(X_{n}\right)\right)} \\
& =\theta\left(\sum_{j=1}^{f(k)}\left\|E_{j}\left(\sum_{i=1}^{f(k)} b_{i} \otimes y_{i}\right)\right\|_{S_{2}\left(X_{n}\right)}^{\frac{1}{2}}\right. \\
& =\theta\left(\sum_{j=1}^{f(k)}\left\|b_{j} \otimes y_{j}\right\|_{S_{2}\left(X_{n}\right)}^{2}\right)^{\frac{1}{2}} \\
& =\theta\left(\sum_{j=1}^{f(k)}\left\|b_{j}\right\|_{S_{2}}^{2}\left\|y_{j}\right\|_{X_{n}}^{2}\right)^{\frac{1}{2}}=\theta\left(\sum_{j=1}^{f(k)}\left\|b_{j}\right\|_{S_{2}}^{2}\right)^{\frac{1}{2}} .
\end{aligned}
$$

The first and the third equality comes from Proposition 2.1 and Lemma 3.6 of [9], respectively.

For the right inequality we will show the following more general results using induction on $n$.

$$
\left\|\sum_{i=1}^{f(k)} b_{i} \otimes y_{i}\right\|_{S_{2}\left(X_{n}\right)} \leq\left(\sum_{i=1}^{f(k)}\left\|b_{i}\right\|_{S_{2}}^{2}\right)^{\frac{1}{2}}
$$

for all $\left(b_{i}\right)_{i=1}^{f(k)} \subseteq \mathcal{K}$ and for any disjoint and normalized sequence $\left(y_{i}\right)_{i=1}^{f(k)}$.

When $n=0$, we have (4.1) since

$$
C B\left(O H, \min \ell_{2}\right)=B\left(O H, \min \ell_{2}\right)
$$

isometrically and $\left(e_{j 1}\right)_{j \geq 1}$ and $\left(y_{j}\right)_{j=1}^{f(k)}$ are orthonormal. 
Now suppose we have (4.1) for $n$. By the induction hypothesis and Proposition 2.1 of [9] we have for any "allowable" sequence $\left\{E_{j}\right\}_{j=1}^{f(l)} \subseteq \mathbb{N}$ that

$$
\begin{aligned}
\theta\left\|\left(E_{j}\left(\sum_{i=1}^{f(k)} b_{i} \otimes y_{i}\right)\right)_{j=1}^{f(l)}\right\|_{S_{2}\left(\ell_{2}^{f(l)}\left(X_{n}\right)\right)} & =\left(\sum_{j=1}^{f(l)}\left\|\sum_{i=1}^{f(k)} b_{i} \otimes \theta E_{j} y_{i}\right\|_{S_{2}\left(X_{n}\right)}^{2}\right)^{\frac{1}{2}} \\
& \leq\left[\sum_{j=1}^{f(l)}\left(\sum_{i=1}^{f(k)}\left\|b_{i}\right\|_{S_{2}}^{2}\left\|\theta E_{j} y_{i}\right\|_{X_{n}}^{2}\right)\right]^{\frac{1}{2}} \\
& =\left[\sum_{i=1}^{f(k)}\left\|b_{i}\right\|_{S_{2}}^{2}\left(\sum_{j=1}^{f(l)}\left\|\theta E_{j} y_{i}\right\|_{X_{n}}^{2}\right)\right]^{\frac{1}{2}} \\
& \leq\left[\sum_{i=1}^{f(k)}\left\|b_{i}\right\|_{S_{2}}^{2}\left\|y_{i}\right\|_{X_{O H}}^{2}\right]^{\frac{1}{2}}=\left[\sum_{i=1}^{f(k)}\left\|b_{i}\right\|_{S_{2}}^{2}\right]^{\frac{1}{2}} .
\end{aligned}
$$

Thus, we have that

$$
e_{i} \mapsto\left(E_{j} y_{i}\right)_{j=1}^{f(l)}, O H_{f(k)} \rightarrow \ell_{2}^{f(l)}\left(X_{n}\right)
$$

is a complete contraction for all "allowable" sequence $\left\{E_{j}\right\}_{j=1}^{f(l)}$, which implies

$$
e_{i} \mapsto y_{i}, O H_{f(k)} \rightarrow X_{n+1}
$$

is also a complete contraction. Consequently, we get the desired induction result for $n+1$.

\section{2. $X_{O H}$ is a nontrivial weak- $O H$ space.}

Proposition 4.5. For $n \in \mathbb{N}$ we consider

$$
Y_{n}=\overline{\operatorname{span}}\left\{t_{i}\right\}_{i \geq n+1} \subseteq X_{O H} .
$$

Then for any $E \subseteq Y_{n}$ with $\operatorname{dim} E=n$, we have

$$
d_{c b}\left(E, O H_{n}\right) \leq 3 \theta^{-1} .
$$

Theorem 4.6. $X_{O H}$ is a weak-OH space.

Lemma 4.7. Let $N \in \mathbb{N}$ be fixed. Then, for any $n \geq 0$ and any $y, z \in X_{O H}$ with suppy $\subseteq\{1,2, \cdots, N\}$ and supp $\subseteq \subseteq\{N+1, N+2, \cdots\}$ we have

$$
\|y+z\|_{n+1} \leq \max \left\{\|y\|_{n+1}+\alpha\|z\|_{n},\|z\|_{n+1}\right\},
$$

where $\alpha=\max \{1, \theta \sqrt{f(N)}\}$.

Proof. Let's fix $n \geq 0$. If $\|y+z\|_{n+1}=\|y+z\|_{n}$, then it is trivial.

Now we consider any "allowable" sequence $\left\{E_{j}\right\}_{j=1}^{f(k)} \subseteq \mathbb{N}$. When $k>N$, we have

$$
E_{j}(y+z)=E_{j} z
$$

so that

$$
\theta\left\|\left(E_{j}(y+z)\right)_{j=1}^{f(k)}\right\|_{\substack{\mathcal{K} \otimes_{\min } \ell_{2}^{f(k)}\left(X_{n}\right) \\ 17}} \leq\|z\|_{n+1} .
$$


When $k \leq N$, we have

$$
\begin{aligned}
& \theta\left\|\left(E_{j}(y+z)\right)_{j=1}^{f(k)}\right\|_{\mathcal{K} \otimes_{\min } \ell_{2}^{f(k)}\left(X_{n}\right)} \\
& \leq \theta\left\|\left(E_{j} y\right)_{j=1}^{f(k)}\right\|_{\mathcal{K} \otimes_{\min } \ell_{2}^{f(k)}\left(X_{n}\right)}+\theta\left\|\left(E_{j} z\right)_{j=1}^{f(k)}\right\|_{\mathcal{K} \otimes_{\min } \ell_{2}^{f(k)}\left(X_{n}\right)} \\
& \leq\|y\|_{n+1}+\theta\left\|\left(E_{j} z\right)_{j=1}^{f(k)}\right\|_{\ell_{2}^{f(k)}\left(\mathcal{K} \otimes_{\min } X_{n}\right)} \\
& =\|y\|_{n+1}+\theta \sqrt{\sum_{j=1}^{f(k)}\left\|E_{j} z\right\|_{n}^{2}} \\
& \leq\|y\|_{n+1}+\theta \sqrt{f(N)}\|z\|_{n} .
\end{aligned}
$$

Lemma 4.8. For any $n \geq 0$ and any $x \in \mathcal{K} \otimes_{\min } X_{O H}$ we have

$$
\|x\|_{\mathcal{K} \otimes_{\min } X_{O H}} \leq\left\|\left(x, \theta^{n} x\right)\right\|_{\mathcal{K} \otimes_{\min }\left(X_{n} \oplus_{2} O H\right)} .
$$

Proposition 4.9. For any $n \geq 0$ and for any infinite dimensional subspace $E \subseteq X_{n}$ we have that $R \otimes_{\min } E$ contains an isomorphic copy of $c_{0}$.

Theorem 4.10. $X_{O H}$ is not completely isomorphic to $O H$.

Proof. The only different point from the proof of Theorem 3.14 is to observe that $R \otimes_{\min } O H$ does not contain any isomorphic copy of $c_{0}$. Indeed, we have

$$
\begin{aligned}
R \otimes_{\min } O H & =O H \otimes_{h} R=[R, C]_{\frac{1}{2}} \otimes_{h} R \\
& =\left[R \otimes_{h} R, C \otimes_{h} R\right]_{\frac{1}{2}} .
\end{aligned}
$$

Since $R \otimes_{h} R$ and $C \otimes_{h} R$ are isometric to $S_{2}$ and $S_{\infty}$, respectively, $R \otimes_{\min } O H$ is isometric to $S_{4}$. Thus, $R \otimes_{\min } O H$ is a reflexive Banach space with a basis, so that it does not contain a isomorphic copy of $c_{0}$.

Remark 4.11. Instead of $X_{0}=\min \ell_{2}$ we can use $R+C$ in the above construction for another nontrivial Hilbertian weak- $O H$ space $Y_{O H}$. However, we do not know $X_{O H}$ and $Y_{O H}$ are completely isomorphic or not at the time of this writing.

Remark 4.12. By a similar procedure we can construct a non-Hilbertian weak- $H$ space $T_{O H}$. The construction is as follows.

We will define a sequence of norms on $\mathcal{K}_{0} \otimes c_{00}$ again. Consider a fixed constant $0<\theta<1$. For $x \in \mathcal{K}_{0} \otimes c_{00}$ we define

$$
\|x\|_{0}^{\prime}=\|x\|_{\mathcal{K} \otimes_{\min } c_{0}}
$$

and for $n \geq 1$ we define

$$
\|x\|_{n+1}^{\prime}=\max \left\{\|x\|_{n}^{\prime}, \theta \sup \left\|\left(E_{j} x\right)_{j=1}^{f(k)}\right\|_{\mathcal{K} \otimes_{\min } \ell_{2}^{f(k)}\left(T_{n}\right)}\right\}
$$

where the inner supremum runs over all "allowable" sequence $\left\{E_{j}\right\}_{j=1}^{f(k)} \subseteq \mathbb{N}$. We denote the completion of $\left(c_{00},\|\cdot\|_{n}^{\prime}\right)$ by $T_{n+1}$, and $T_{n+1}$ inherits the operator space structure from $T_{n} \oplus_{\infty} \ell_{\infty}\left(I ;\left\{\ell_{2}^{f(k)}\left(T_{n}\right)\right\}\right)$, where $I$ is the collection of all allowable sequences.

Then by a similar argument as in Proposition 4.1 we can show that

$$
\|x\|_{\mathcal{K}_{\min c_{0}}} \leq\|x\|_{n}^{\prime} \leq\|x\|_{\mathcal{K} \otimes_{\min O H}}
$$

for all $n \geq 0$ and $\left(\|x\|_{n}^{\prime}\right)_{n \geq 0}$ is increasing. Thus,

$$
\|x\|^{\prime}=\lim _{n \rightarrow \infty}\|x\|_{n}^{\prime}
$$


converges for all $x \in \mathcal{K}_{0} \otimes c_{00}$, so that $T_{O H}$, the completion of $\left(c_{00},\|\cdot\|^{\prime}\right)$ inherits the operator space structure from $\ell_{\infty}\left(T_{n}\right)$.

If we look at the underlying Banach space of $T_{O H}$, then it is nothing but a variant of modified 2-convexification of Tsirelson's space in (2) of Notes and Remarks in X.e (p.117 of [1]). The only difference is the fact that we replaced $f(k)=k$ into $f(k)=\left(4 k^{3}\right)^{k}$, and it is well-known that our $T_{O H}$ is isomorphic to (as a Banach space) the 2-convexified Tsirelson's space (see X.e. and Appendix b. in [1), which is not isomorphic to any Hilbert space.

By a similar argument we can show $T_{O H}$ is a non-Hilbertian example of weak- $O H$ space.

\section{REFERENCES}

1. P. G. Casazza; T. J. Shura, Tsirelson's space. Lecture Notes in Mathematics, 1363. SpringerVerlag, Berlin, 1989.

2. J. Diestel; H. Jarchow; A. Tonge, Absolutely summing operators. Cambridge Studies in Advanced Mathematics, 43. Cambridge University Press, Cambridge, 1995.

3. E. G. Effros; Z. J. Ruan; Operator spaces. London Mathematical Society Monographs. New Series, 23. The Clarendon Press, Oxford University Press, New York, 2000.

4. H. H. Lee, Type and cotype of operator spaces, preprint, 2006.

5. H. H. Lee, Weak type $(2, H)$ and weak cotype $(2, H)$ of operator spaces, preprint, 2006.

6. G. Pisier, Weak Hilbert spaces. Proc. London Math. Soc. (3) 56(1988), no. 3, 547-579.

7. G. Pisier, The volume of convex bodies and Banach space geometry. Cambridge Tracts in Mathematics, 94. Cambridge University Press, Cambridge, 1989.

8. G. Pisier, The operator Hilbert space OH, complex interpolation and tensor norms. Mem. Amer. Math. Soc. 122 (1996), no. 585.

9. G. Pisier, Non-commutative vector valued $L_{p}$-spaces and completely $p$-summing maps. Astérisque(Soc. Math. France) 247(1998), 1-111.

10. G. Pisier, Introduction to operator space theory. London Mathematical Society Lecture Note Series, 294. Cambridge University Press, Cambridge, 2003.

11. N. Tomczak-Jaegermann, Banach-Mazur distances and finite-dimensional operator ideals. Pitman Monographs and Surveys in Pure and Applied Mathematics, 38. Longman Scientific \& Technical, 1989.

Department of Mathematical Sciences, Seoul National University San56-1 ShinrimDONG KWANAK-GU SEOUl 151-747, Korea

E-mail address: Lee.hunhee@gmail.com, bbking@amath.kaist.ac.kr 